\title{
EXPERIENCE REPORT OF PSYCHOSOCIAL CARE TO A PATIENT WITH BREAST CANCER
}

Yêda Maria Silva Peixoto', Márcia de Faria Veloso², Ruffo Freitas-Júnior²

${ }^{1}$ Faculdade Estácio de Sá de Goiás - Goiânia (GO), Brazil.

¿Universidade Federal de Goiás - Goiânia (GO), Brazil.

Introduction: This study presents the experience of psychological care to women with breast cancer, attended at the Advanced Breast Diagnosis Center (CORA), Hospital das Clínicas - UFG, under supervision. Objective: The aim of this study is to describe the phases of greatest psychosocial impact in the treatment of patients and family members. Methodology: The service to EAS was started in November 2017 after, 43 years old, divorced, and provider of three minor children, receiving the diagnosis of phylloid tumor, intense anguish, fear of death, emotional lability, conflicts family and socioeconomic status, presenting a distorted perception of identity. She was mastectomized and underwent chemotherapy and a new surgery in 2018, after a recurrence of sarcoma in the sternum bone, at which point she went into palliative care. Psychological, digital, and social-technical resources were used until her death in May 2020. Results: An improvement was observed in the coping strategy, resolution of family conflicts, recovery of affective bonds and their identity, with the distribution of tasks of the children, and improvement of the family dialogue, including the desire to grant the guardianship of the children to her brother. Discussion: It is necessary to have a systemic look at the patient who arrives at the public hospital. Considerations: This experience was enriching due to the relevance of the role of psychology and its interventions with the multidisciplinary team, promoting a significant improvement in the psychological well-being and quality of life of the patient who arrives with real and imagined suffering aggravated by the disease and treatment.

Keywords: Psychosocial Intervention; Breast Cancer; Palliative Care. 